\title{
THE CHARACTERISTIC ROOTS OF A MATRIX*
}

BY E. T. BROWNE

1. Introduction. If $A$ is a square matrix of order $n$ and $I$ is the unit matrix, the equation in $\lambda$ obtained by equating to zero the determinant $|A-\lambda I|$ is called the characteristic equation of $A$. The roots of this equation are called the characteristic roots of $A$. Although it is not possible to make any definite statement as to the nature of the characteristic roots of the general algebraic matrix $A$, several authors have given upper limits to the roots. The first upper limit seems to have been given by Bendixson $\dagger$ in 1900 .

Let us denote by $A^{\prime}$ and $\bar{A}$ the transpose and the conjugate imaginary, respectively, of the square matrix $A$. If we write

$$
B=\frac{A+\bar{A}^{\prime}}{2}, \quad C=\frac{A-\bar{A}^{\prime}}{2 i},
$$

it is obvious that $\bar{B}^{\prime}=B$ so that $B$ is Hermitian (or real symmetric if $A$ is real). Similarly, $C$ is Hermitian (or skew-symmetric if $A$ is real). Bendixson's theorem then is as follows:

Bendixson's TheOREm. (a) If $\alpha+i \beta$ is a characteristic root of a real matrix $A$ and if $\rho_{1} \geqq \rho_{2} \geqq \cdots \geqq \rho_{n}$ are the characteristic roots (all real) of the symmetric matrix $B=\left(A+A^{\prime}\right) / 2$, then

$$
\rho_{1} \geqq \alpha \geqq \rho_{n} .
$$

(b) If $g^{\prime \prime}$ is the greatest of the numerical values of the elements $\left|\left(a_{i j}-a_{j i}\right) / 2\right|$ of the real skew-symmetric matrix $\left(A-A^{\prime}\right) / 2$, then

$$
|\beta| \leqq g^{\prime \prime}[n(n-1) / 2]^{1 / 2} .
$$

The extension to the case where the elements of $A$ are complex was made in 1902 by Hirsch $\ddagger$ who proved the following theorem:

* Presented to the Society, September 11, 1930.

$\dagger$ Bendixson, Sur les racines d'une équation fondamentale, Acta Mathematica, vol. 25 (1902), pp. 359-365.

$\ddagger$ Hirsch, Acta Mathematica, vol. 25 (1902), pp. 367-370. 
Hirsch's TheOREM. (a) If $\alpha+i \beta$ is a characteristic root of any square matrix $A$ and if we designate by $g$ the greatest of $\left|a_{i j}\right|, *$ by $g^{\prime}$ the greatest of $\left|\left(a_{i j}+\bar{a}_{j i}\right) / 2\right|$, and by $g^{\prime \prime}$ the greatest of $\left|\left(\bar{a}_{i j}-a_{j i}\right) / 2\right|$, then always

$$
\begin{aligned}
|\alpha+i \beta| & \leqq n g, \\
|\alpha| & \leqq n g^{\prime}, \\
|\beta| & \leqq n g^{\prime \prime} .
\end{aligned}
$$

(b) If $\rho_{1}$ is the greatest and $\rho_{n}$ the least (algebraically) of the characteristic roots of $B=\left(A+\bar{A}^{\prime}\right) / 2$, then always

$$
\rho_{1} \geqq \alpha \geqq \rho_{n} \text {. }
$$

In 1904 Bromwich $\dagger$ gave a proof of Hirsch's Theorem (a) and (b) and further extended (b) as follows:

Bromwich's THEOREM. If $\alpha+i \beta$ is a characteristic root of a matrix $A$ and if we denote by $\pm \mu_{1}, \cdots, \pm \mu_{v}(2 v \leqq n)$ the nonzero characteristic roots of the matrix $C=\left(A-\bar{A}^{\prime}\right) /(2 i)$, then $|\beta|$ cannot exceed the greatest of the $\left|\mu_{i}\right|$.

In 1922, for a real matrix $A$, Pick $\ddagger$ gave a proof of Bendixson's Theorem (a) with Bromwich's extension and he showed that (2) can be replaced by $|\beta|=g^{\prime \prime}|\operatorname{ctn} \pi /(2 n)|$ which in general gives a more restricted limit than (2).

In 1927 the author $\S$ attacked the problem from a different angle and proved the following theorem:

If $\lambda$ is a characteristic root of a square matrix $A$ and if $\rho_{1} \geqq \rho_{2} \geqq \cdots \geqq \rho_{n}$ are the characteristic roots (all $\geqq 0$ ) of $A \bar{A}^{\prime}$, then $\rho_{1} \geqq \lambda \bar{\lambda} \geqq \rho_{n}$.

It is the purpose of this paper to show by a very simple method that Hirsch's limits (3), (4) and (5) may be replaced

* Here $\left|A_{i j}\right|$ denotes not the determinant of the matrix $A$ but the absolute value of the number $A_{i j}$.

$\dagger$ Bromwich, On the roots of the characteristic equation of a linear substitution, Acta Mathematica, vol. 30 (1906), pp. 295-304.

¥ Pick, Über die Wurzeln der charakteristischen Gleichung von Schwingungsproblemen, Zeitschrift für angewandte Mathematik und Mechanik, vol. 2 (1922), pp. 353-357.

$\S$ The characteristic equation of a matrix, this Bulletin, vol. 34 (1928), pp. 363-368. 
by limits which never exceed the former and are in general more restricted.

2. The Characteristic Roots of $A \bar{A}^{\prime}$. If $A$ is any square matrix, real or complex, Autonne* has shown that there exist two unitary (real orthogonal, if $A$ is real) matrices $P, Q$ such that

$$
A=\bar{P}^{\prime} N Q
$$

where $N$ has real positive (or zero) numbers in the main diagonal and zeros elsewhere. On forming the product $A \bar{A}^{\prime}$ $=\bar{P}^{\prime} N^{2} P$ it becomes evident that the numbers in the diagonal of $N$ are the positive (or zero) square roots $\rho_{1}^{1 / 2}, \cdots, \rho_{n}{ }^{1 / 2}$ of the characteristic roots of $A \bar{A}^{\prime}$. From (6), $N=P A \bar{Q}^{\prime}$, so that if we denote by $p_{i j}, n_{i j}$ and $q_{i j}$ the element in the $i$ th row and the $j$ th column of $P, N$ and $Q$, respectively, we have

$$
n_{i j}=\sum_{r, s}^{1, \cdots, n} p_{i r} a_{r s} \bar{q}_{j s} .
$$

That is, the elements of $N$ are of the form $\sum_{i j}^{1, \cdots, n} a_{i j} x_{i} y_{j}$ where $\left(x_{1}, \cdots, x_{n}\right)$ and $\left(y_{1}, \cdots, y_{n}\right)$ are sets of numbers such that $\sum_{i} x_{i} \bar{x}_{i}=\sum_{i} y_{i} \bar{y}_{i}=1$.

3. An Upper Limit to the Roots of $A$. Let us denote by $\eta_{i}$ and $\zeta_{i}$ the absolute values of $x_{i}$ and $y_{i}$, respectively. Then the sets $\left(\eta_{1}, \cdots, \eta_{n}\right)$ and $\left(\zeta_{1}, \cdots, \zeta_{n}\right)$ are real sets such that $\sum \eta_{i}{ }^{2}=\sum \zeta_{i}{ }^{2}$ $=1$. Since $\eta_{i}$ and $\zeta_{i}$ are real

$$
\eta_{i} \zeta_{j} \leqq \frac{1}{2}\left(\eta_{i}^{2}+\zeta_{\eta^{2}}\right)
$$

Hence

$$
\begin{aligned}
\left|n_{i i}\right|=\left|\sum a_{i j} x_{i} y_{j}\right| \leqq & \sum\left|a_{i j}\right|\left|x_{i}\right|\left|y_{j}\right| \\
& =\sum\left|a_{i j}\right| \eta_{i} \zeta_{j} \leqq \frac{1}{2} \sum\left|a_{i j}\right|\left(\eta_{i}{ }^{2}+\zeta_{j}^{2}\right) .
\end{aligned}
$$

If $S_{i}$ denotes the sum of the absolute values of the elements in the $i$ th row of $A$ and if $S$ is the greatest of the $S_{i}$, we have

* Autonne, Sur les matrices hyfohermitiennes et les unitaires, Comptes Rendus, vol. 156 (1913), pp. 858-860, in which the theorem is given without proof. In fact the theorem follows as a consequence of the author's Theorem IV on p. 367 of the aforementioned paper. See also Taber, On the linear transformations between two quadrics, Proceedings of the London Mathematical Society, vol. 24 (1892-93), pp. 290-306, in which the theorem for $A$ real and non-singular is contained implicitly. 


$$
\sum_{i, j}\left|a_{i j}\right| \eta_{i}{ }^{2}=\sum_{i} \eta_{i}{ }^{2} \sum_{j}\left|a_{i j}\right|=\sum_{i} \eta_{i}{ }^{2} S_{i} \leqq S \sum \eta_{i}{ }^{2}=S .
$$

Similarly, if $T_{i}$ denotes the sum of the absolute values of the elements in the $i$ th column of $A$ and if $T$ is the greatest of the $T_{i}$,

Hence

$$
\sum_{i, j}\left|a_{i j}\right| \zeta_{j}^{2} \leqq T
$$

$$
\left|n_{i i}\right|=\rho_{i}{ }^{1 / 2} \leqq(S+T) / 2 .
$$

Now from the author's theorem as quoted earlier in the paper, if $\lambda$ is a characteristic root of $A$ and $M$ is the greatest of the $\rho$ 's, then

$$
|\lambda| \leqq M^{1 / 2}
$$

Hence

$$
|\lambda| \leqq(S+T) / 2 .
$$

We therefore have the following theorem:

THEOREM. If $S_{i}\left(T_{i}\right)$ is the sum of the absolute values of the elements in the ith row (column) of a square matrix $A$ and if $S(T)$ is the greatest of the $S_{i}\left(T_{i}\right)$, the absolute value $|\lambda|$ of a characteristic root $\lambda$ of $A$ cannot exceed $(S+T) / 2$.

Equation (3) of Hirsch's Theorem (a) obviously follows as a corollary to this theorem. It is clear that the limit given by the latter can never exceed that given by Hirsch's criterion and is in general less.

The limit is sometimes actually attained, for example, if $A$ is a matrix each of whose elements is the square of the corresponding element of a real orthogonal matrix. In this case $A$ obviously has the characteristic root +1 . Moreover, the limit is always attained if $A$ is a circulant, (that is, $a_{i j}=a_{j-i+1}$, for $i \leqq j$; $a_{i j}=a_{n+i-i+1}$ for $i>j$ ) whose elements are real and $\geqq 0$.

In particular, if $A$ is Hermitian, $S_{i}=T_{\imath}$ and hence $S=T$, so that we have the following corollary.

Corollary 1. If $S_{i}$ is the sum of the absolute values of the elements in the ith row of an Hermitian matrix $A$, and if $S$ is the greatest of the $S_{i}$, the numerical value of a characteristic root $\lambda$ of $A$ cannot exceed $S$. 
The limit given in the corollary is evidently attained if $A$ consists entirely of zeros except in the diagonal. It is also attained if $A$ is a real cyclic matrix, (that is, $a_{i j}=a_{k}$ where $k$ is the least positive residue of $i+j-1, \bmod n)$ with positive or zero elements.

On making use of Hirsch's Theorem (b) we have also the following corollary.

Corollary 2. If $\alpha+i \beta$ is a characteristic root of $A$ and if $S_{j}{ }^{\prime}$ is the sum of the absolute values of the elements in the jth row of the Hermitian matrix $B=\left(A+\bar{A}^{\prime}\right) / 2$, then if $S^{\prime}$ is the greatest of the $S_{j}{ }^{\prime}$, it follows that $|\alpha| \leqq S^{\prime}$.

Equation (4) of Hirsch's Theorem follows directly from this corollary. Moreover, it is clear that our criterion usually gives a narrower limit than that of Hirsch.

By invoking Bromwich's Theorem we can state also the following corollary.

Corollary 3. If $\alpha+i \beta$ is a characteristic root of $A$ and if $S_{j}{ }^{\prime \prime}$ is the sum of the absolute values of the elements in the jth row of the Hermitian matrix $C=\left(A-\bar{A}^{\prime}\right) /(2 i)$, then if $S^{\prime \prime}$ is the greatest of the $S_{j}{ }^{\prime \prime}$, we have $|\beta| \leqq S^{\prime \prime}$.

Equation (5) of Hirsch's Theorem (a) follows directly from this corollary. Corollaries 2 and 3 , which have been deduced from our theorem, might have been proved directly without invoking Autonne's theorem. For if we write, as in $\$ 1, B=$ $\left(A+\bar{A}^{\prime}\right) / 2, C=\left(A-\bar{A}^{\prime}\right) /(2 i)$, it is clear that $A=B+i C$ where $B$ and $C$ are Hermitian matrices. Suppose now that $\alpha+i \beta$ is a characteristic root of $A$. Then there exists a set $\left(x_{1}, \cdots, x_{n}\right)$ $\neq(0, \cdots, 0)$, and which we may suppose to have been divided through by the proper non-vanishing factor so that $\sum x_{i} \bar{x}_{i}=1$, such that

$$
\sum_{j} a_{t j} x_{j}=\sum_{j} b_{t j} x_{j}+i \sum_{j} c_{t j} x_{j}=(\alpha+i \beta) x_{t} \quad(t=1, \cdots, n) .
$$

On multiplying these equations through by $\bar{x}_{t}$ and summing as to $t$, we have

$$
\sum_{t, j} b_{t j} \bar{x}_{t} x_{j}+i \sum_{t, j} c_{t j} \bar{x}_{t} x_{j}=\sum_{t}(\alpha+i \beta) \bar{x}_{t} x_{t}=\alpha+i \beta .
$$


Now since $B$ and $C$ are Hermitian matrices each of the summations on the left is real. Hence, equating real and imaginary parts in (10) we have*

$$
\begin{aligned}
\alpha & =\sum_{i, j} b_{i j} \bar{x}_{i} x_{j}, \\
\beta & =\sum_{i, j} c_{i j} \bar{x}_{i} x_{j} .
\end{aligned}
$$

If now we denote by $\eta_{i}$ the absolute value of $x_{i}$ and proceed as in (9), Corollaries 2 and 3 follow at once.

The University of North Carolina

\title{
ON THE REDUCTION OF THE INDEFINITE BINARY QUADRATIC FORMS $\dagger$
}

\author{
BY J. V. USPENSKY
}

The reduction theory of the indefinite binary forms has been presented in widely different forms and from various points of view. But whatever point of view is adopted, it seems that Hermite's principle of continuous variables under more or less disguised form constitutes an essential foundation of all the existing theories of reduction.

Hermite's principle in its simplest aspect consists in association with a given indefinite form of a positive quadratic form containing a continuously varying positive parameter and the study of integral values of variables which give successive minima of the latter. However, the reduced forms in Hermite's theory differ from those in the classical Gaussian theory of reduction. A little contribution to the theory of reduction which this article contains has for its purpose to show how, by substituting for Hermite's positive quadratic form a certain nonhomogeneous function containing a variable positive parameter, we obtain precisely the Gaussian reduced forms.

Let

$$
\xi=\alpha x+\beta y, \quad \eta=\gamma x+\delta y
$$

* See Hirsch, loc. cit., p. 369.

$\dagger$ Presented to the Society, April 5, 1930. 